\title{
A Low-Power Ultra-Fast Capacitor-Less LDO with Advanced Dynamic Push-Pull Techniques
}

\author{
Xin Ming, Ze-kun Zhou, and Bo Zhang \\ State Key Laboratory of Electronic Thin Films and Integrated Devices, \\ University of Electronic Science and Technology of China, \\ 610054 Chengdu, China \\ \{mingxin, zkzhou, zhangbo\} @uestc.edu.cn
}

\begin{abstract}
A current-efficient, capacitor-less low-dropout regulator (LDO) with fast-transient response for portable applications is presented in this chapter. It makes use of an adaptive biasing common-gate amplifier to extend loop bandwidth of the LDO at heavy loads greatly. Also, the dynamic push-pull (DPP) slew-rate enhancement (SRE) circuit based on capacitive coupling detects rapid voltage spikes at the output to provide an extra current to charge and discharge the large gate capacitance of the power transistor momentarily. The proposed circuit has been implemented in a $0.35 \mu \mathrm{m}$ standard CMOS process. Experimental results show that it can deliver $100 \mathrm{~mA}$ load current at $150 \mathrm{mV}$ dropout voltage. It only consumes $10 \mu \mathrm{A}$ quiescent current at no-load condition and is able to recover within $0.8 \mu$ s even under the maximum load current change.
\end{abstract}

Keywords: adaptive biasing, dynamic push-pull technique, capacitive coupling, slew-rate enhancement, high bandwidth, low-dropout regulator, system-on-chips.

\section{Introduction}

Various multimedia and portable devices lead the trend of system-on-chip (SoC) integration. The power management is an essential part in the battery-powered system. To get a fast transient response and noise-less output supply voltage, the onchip capacitor-less LDO is demanded to be integrated with the SoC systems. However, it takes the restriction of minimum load current and slow transient response into design consideration due to the low supply voltage [1]-[4].

Normally, transient response is a critical dynamic specification in LDO design, which is dominated by the loop-gain bandwidth and slew rate at the gate of the power transistor. Both the amplitude of voltage spike and recovery time of regulated output voltage will affect its overall accuracy. Unfortunately, the generic approaches to optimize the transient response using external capacitors and large bias current are no longer useful in the power-saving SoC application. Several techniques are thus proposed to improve the transient responses without increasing quiescent current so much [5]-[9]. An active-frequency compensation circuit is introduced in [5] to greatly boost the effective current multiplication factor by at least one order of magnitude and 
extend the loop bandwidth drastically. A low-power fast-transient low-dropout regulator with multiple small-gain stages is employed in [6] to provide loop gain enhancements without introducing low-frequency poles before the unity-gain frequency, which leads to larger loop gain and bandwidth. An adaptive reference control technique is proposed in [7] by dynamically and smoothly adjusting the reference voltage so as to increase the slew rate of error amplifier (EA). A low-power analog driver based on a single-stage amplifier with an embedded current-detection SRE circuit is presented in [8]. A low-dropout linear regulator topology with replicabiased common-source unity-gain buffer is used in [9] to overcome the bandwidth limitation of the feedback loop.

Recently, non-static biasing has been proved to be an effective way to improve transient responses in low-power design, which enables bias current to be dramatically increased for bandwidth extension and slew-rate improvement. Adaptive biasing $(\mathrm{AB})$, that increases the bias current according to the magnitude of the output current, is employed in [10]-[12]. For example, the buffer stage is adaptively biased as shown in [10], where the increase in current in the buffer stage aids the circuit by pushing the parasitic pole associated with parasitical capacitors at the gate of power transistors to higher frequencies and by increasing the current available for slew-rate conditions; one more choice is used at the input stage in [11] and [12] to simultaneously extend both slewing and bandwidth. Another more current-efficient way is to utilize dynamic biasing technique where more bias current is adopted only at the transient instant when the output current is changed [13]. Based on this idea, the capacitive coupling and dynamic charging method is reported lately, which is very promising in increasing circuitry response speed while keeping static power consumption low. It can be utilized to increase the bias current of error amplifier momentarily [14]-[18] or construct a current-boosting voltage buffer [19] and a differentiator [20] for bandwidth and slew-rate improvement. Slewing detection can be made by either monitoring an internal node or at the output. The important issue here is that when the capacitive coupling circuit is used in a closed loop scheme, since it will introduce other control loop after being triggered when the capacitive coupling circuit is used in a closed loop scheme, the transient stability analysis is getting more complicated, which is very necessary to make sure it works robustly.

In this work, an adaptive biasing error amplifier with a low-power dynamic pushpull SRE circuit is applied to a capacitor-less LDO to show enhancements in transient responses. This chapter is organized as follows. Concept of the proposed LDO is discussed in Section 2. Circuit design and implementation are shown in Section 3. Experimental results and conclusions are given in Sections 4 and 5, respectively.

\section{Design of the Proposed Circuits}

As shown in Fig. 1, the basic structure of this ultra-fast capacitor-less LDO is similar with [13] focusing on dynamic biasing. It is constructed by two differential commongate transconductance cells, a voltage buffer, a current-summation circuit and an additional SRE circuit. The two $G_{m}$ cells, which are made basically by a pair of matched transistors $\left(M_{a}\right.$ and $M_{b}$ in Fig. 1 as an example) in the form of a current mirror, are cross-coupled achieving a push-pull output stage to inject and withdraw 
more current for charging and discharging during the transient instant. Because the output current $I_{o}$ has a quadratic dependence on its input-voltage difference according to the square-law characteristic of MOS transistor, the maximum output current $I_{\text {omax }}$ is no longer limited by the constant-current source as in the case of conventional amplifier with a tail-current. This is very practical for fully on-chip LDOs to improve transient response since low power and high slew rate can be realized at the same time.

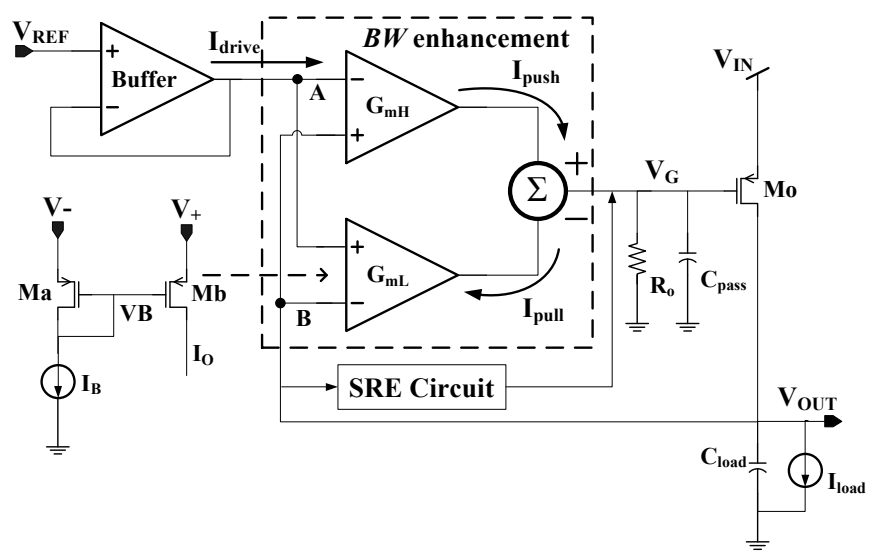

Fig. 1. Conceptual schematic of the proposed capacitor-less LDO regulator

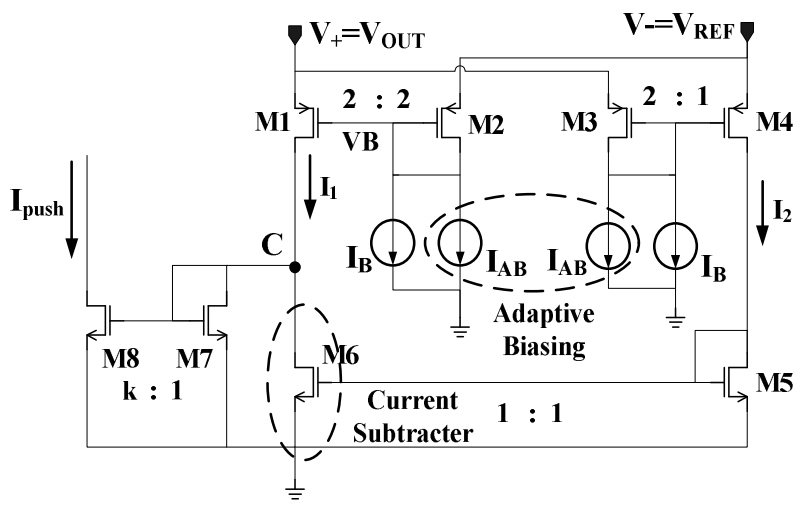

Fig. 2. Principle of the high bandwidth transconductance amplifier $G_{m H}$

Although the SR-limit problem has been improved by enabling a higher bias current during the transient instant, this differential common-gate amplifier has limited input common-mode range (ICMR) and, most importantly, limited bandwidth which is determined by transconductance $G_{m}$ and gate capacitance $C_{\text {pass }}$ of the power transistor as shown in Fig. 1. Therefore, fast changing voltage spike cannot be detected effectively by the amplifier at low bias current. Moreover, this approach is 
not applicable when $V_{\text {OUT }}$ is at a small value, which happens when providing an adaptive supply for a power-saving SoC design. To combat the aforementioned challenges, a current efficient and high bandwidth error amplifier with a dynamic push-pull SRE circuit is proposed in this chapter.

\subsection{Adaptive Biasing Error Amplifier}

It is obvious that a larger transconductance $G_{m}$ means a larger GBW and faster transient response at the gate of the power transistor, which requires more power applied to the LDO. However, the traditional methods to improve $G_{m}$ of this simple common-gate amplifier have some difficulties to be realized. For instance, a large bias current $I_{B}$ will increase the minimum input voltage for the amplifier, deteriorating ICMR; also increasing the aspect ratios of input transistors may put them into weakinversion region when a low quiescent current has been adopted. Here, a currentmode method called current subtracter can be utilized to resolve this problem. As shown in Fig. 2, another duplicated common-gate amplifier $\left(\mathrm{M}_{3}-\mathrm{M}_{4}\right)$ has been crosscoupled with $\mathrm{M}_{1}-\mathrm{M}_{2}$ to provide a current $I_{M 4}$ that varies contrarily compared to $I_{M 1}$ and is redirected to node $\mathrm{C}$. The only difference is the aspect ratio of input transistors, which is $2 / 1$, to guarantee a normal bias point of the total output current $I_{\text {push }}$. The larger a voltage pike $\Delta V_{+}$is, the more $I_{p u s h}$ will be gained compared to using $\mathrm{M}_{1}-\mathrm{M}_{2}$ alone. As a result, the total transconductance $G_{m}$ is enhanced by a factor of 1.5 as given by

$$
G_{m}=k\left(g_{m 1}+g_{m 4}\right)=1.5 k g_{m 1}
$$

where $k$ is the gain of current mirror $\mathrm{M}_{7}-\mathrm{M}_{8}$ and set to be 3 in the circuit for boosting output-driving ability. This structure has nearly the same effects as increasing $I_{B}$ to increase $G_{m}$ while introducing less pressure on ICMR design.

Note that the power transistor can be designed to work in linear region when heavy load occurs, such that a more efficient usage of the chip area is achieved. In saturation region, the relationship between $I_{d}$ and $V_{g s}$ is quadratic, and in linear region, it is linear, where an equal factor of increment in $I_{d}$ requires a larger increment of $V_{g s}$. Therefore, the circuit needs larger bandwidth and slew rate at heavy load for high speed control, which is achieved successfully by adaptive biasing [9]. The operation revolves around sensing the output current of the regulator and feeding back a ratio of the current to the input stage of the amplifier. This can be done by a simple current mirror and a sense MOSFET that are area efficient. In addition to the small fixed biasing current $I_{B}$, a feedback current $I_{A B}$ relating to load current $I_{\text {load }}$ (i.e., $I_{A B}=\beta I_{\text {load }}$ ) is applied to the drain of transistor $\mathrm{M}_{2}$ to control $V_{g s 2}$ at different loads. Because $V_{g s 1}$ and $V_{g s 2}$ are equal at DC operating point, the transconductance $g_{m 1}$ of $\mathrm{M}_{1}$ can be expressed as

$$
g_{m 1}=\sqrt{2 \mu_{p} C_{o x}(W / L)_{1}\left(I_{B}+I_{A B}\right)}
$$

The resulting larger bias current at heavy loads increases transconductance of the input pair, leading to a larger bandwidth of the amplifier. During low load current 
conditions, the feedback current $I_{A B}$ is negligible, yielding a high current efficiency and not aggravating battery life.

One important design issue is about carefully setting $I_{A B}$ or aspect ratio $\beta$ between the current-sense transistor and power MOSFET at different loads. Too small $\beta$ will not gain dynamic biasing advantages; however, since $\mathrm{M}_{2}$ is diode-connected and the input $V$. is a stable reference voltage, too large $\beta$ will introduce more feedback current $I_{A B}$ to the input stage pushing $V_{B}$ to a very low voltage especially when $V_{O U T}$ is small, which may result in transistors in the current source of $I_{B}$ and $I_{A B}$ entering into linear region. If this unluckily happens at large load current, there exists no isolation between ground and bias voltage $V_{B}$. The ground noises will couple freely to the gate of input transistors, degrading performances of the amplifier. In this circuit, the largest load current is $100 \mathrm{~mA}$ and the aspect ratio of $I_{\text {load }} I_{A B}$ is chosen as $10000 / 1$, where the largest feedback current is approximately $10 \mu \mathrm{A}$.

\subsection{Dynamic Push-Pull SRE Circuit}

Unluckily, the adaptive biasing is activated only when the gate voltage of the power $\operatorname{MOS}\left(V_{G}\right)$ goes down (i.e., when the feedback is going to compensate an abrupt increment of load current). However, if the load current suddenly increases, an amount of time occurs before $V_{G}$ is moved down and before the adaptive biasing is activated, which is determined by the bandwidth of the loop. This latency may strongly reduce effectiveness of the adaptive biasing. For example, when the load current steps down from heavy load to light load, the fast charging of the pass transistor gate enables small overshoot and fast recovery of the LDO output voltage. However, the biasing current of the amplifier is low at light load that leads to a large undershoot of the output voltage when the load current steps up from the minimum to the maximum in a very short time.

1) Principle of Operation: In order to get rid of the dependence on limited bandwidth and reduce output voltage spikes and recovery time further, a SR enhancement (SRE) circuit based on dynamic push-pull (DPP) techniques is implemented in parallel with the $\mathrm{AB}$ error amplifier to get a better regulated power supply. The SRE circuit only provides a dynamic current to charge or discharge gate capacitance $C_{\text {pass }}$ of the power transistor during transient if large voltage spikes take place, and is completely turned off in the static state, dissipating small quiescent current. It should improve both loopgain bandwidth and slew rate at the gate drive of power transistor, while maintaining high current efficiency in static state.

Normally, the SRE circuit consists of a sensing and driving circuit [21]-[23]. How to avoid a larger loading capacitance due to additional structures as well as high dynamic current at input stage in these existing methods is critical. For example, a current-detection SRE circuit detecting changes in the current signal at active load of the core amplifier is reported in [8]. The advantage is that it does not increase the loading of error amplifier. 


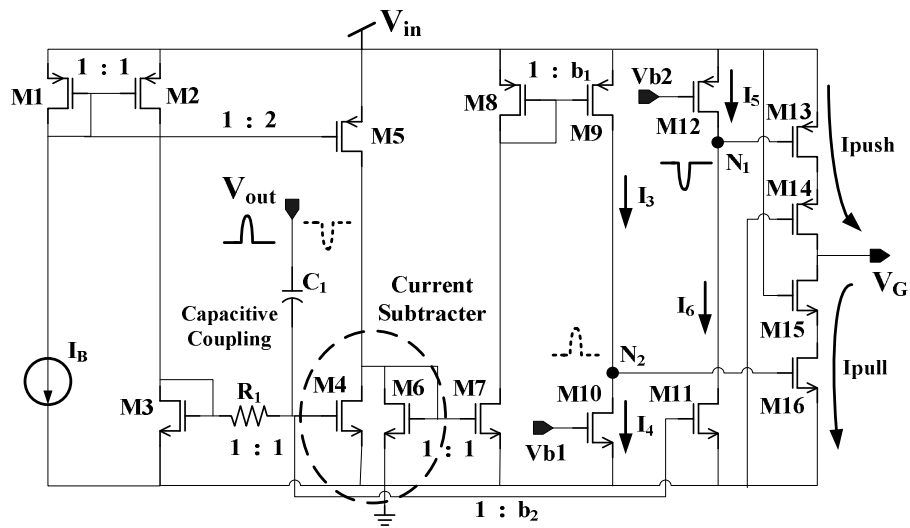

(a)

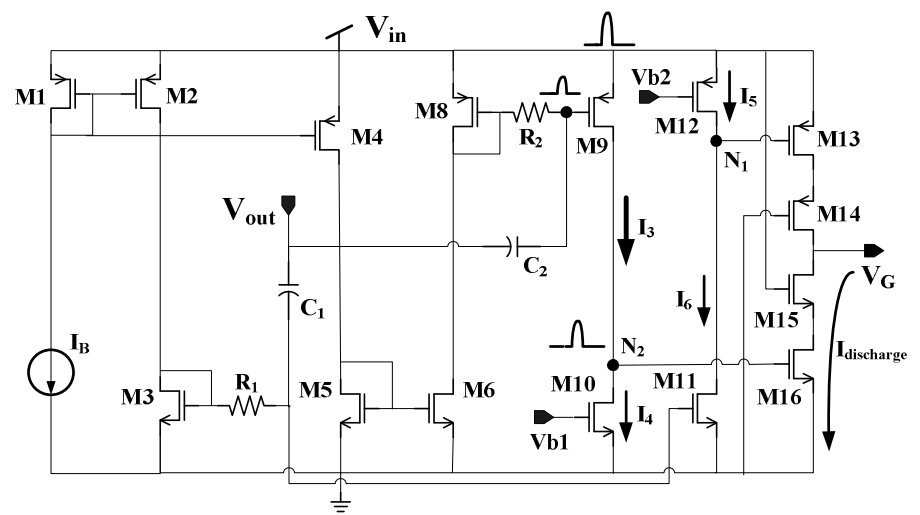

(b)

Fig. 3. DPP SRE circuits with capacitive coupling (a) Proposed DPP techniques (b) DPP structure with two coupling capacitors

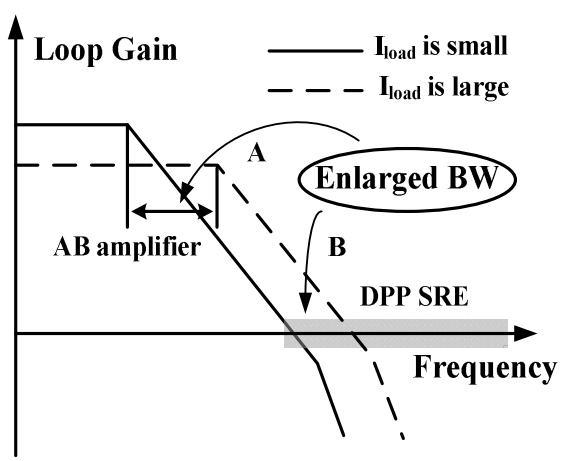

Fig. 4. Sketch map of improvements for the loop bandwidth 
In the proposed structure shown in Fig. 3(a), the sensing circuit adopts a voltage detection method based on capacitive coupling. It senses rapid transient voltage changes at the output of the LDO and then changes current signal $I_{M 4}$ or $I_{M 11}$ to trigger a dynamic push-pull circuit for increasing the driving current momentarily. The basic circuit is a modification to current mirrors $\mathrm{M}_{3}-\mathrm{M}_{4}$ and $\mathrm{M}_{3}-\mathrm{M}_{11}$, where capacitor $C_{1}$ and resistor $R_{1}$ have been added to realize a high-pass filter. It provides a fast path to detect the output voltage spikes. As shown by the timing diagrams in Fig. 3(a), when the amplitude of $V_{\text {OUT }}$ changes from low to high $(\Delta V)$ instantaneously (represented by the real line), the rapid voltage change couples to the gate of $\mathrm{M}_{11}$ directly due to the high-pass property of $C_{1}$. When $C_{1}$ is chosen to be much larger than $C_{g s 3}+$ $C_{g_{s}}+C_{g_{s 11}}$, the gate voltage of $\mathrm{M}_{11}$ is dominated by the coupled signal from $C_{1}$ in this instant. Thus, $V_{g s 11}$ is changed momentarily and the extra current $\Delta I_{6}$ can be found from [14]

$$
\Delta I_{6} \approx g_{m 11} \Delta V=\sqrt{2 b_{2} I_{B} \mu_{n} C_{o x}(W / L)_{M 11}} \Delta V
$$

It is found that a larger aspect ratio of the current mirror helps to increase $\Delta I_{6}$ for injecting more transient current, but at the penalty of increased quiescent current in steady state. Therefore, the size of $\mathrm{M}_{11}$ should be carefully designed to strike a balance between the above tradeoffs. This consideration is also applicable to transistor size design of $\mathrm{M}_{4}$. When $V_{\text {OUT }}$ changes from high to low (represented by the dotted line), the coupling effect generates a smaller $I_{M 4}$ and triggers the pull action. When $V_{\text {OUT }}$ stays at a constant voltage in the steady state, $C_{1}$ is open-circuited, resulting in an auto shutdown of the current boosting circuit. Besides, this coupling effect is independent of the DC value of $V_{O U T}$ due to the high-pass characteristic of $C_{1}$, so the proposed method is suitable for detecting any output voltage level, improving ICMR of the amplifier considerably.

The driving circuit is composed of transistors $\mathrm{M}_{9}-\mathrm{M}_{16}$. Based on the appropriate ratios of current mirror $\left(b_{1}, b_{2}\right), \mathrm{M}_{9}$ and $\mathrm{M}_{10}\left(\mathrm{M}_{11}\right.$ and $\left.\mathrm{M}_{12}\right)$ are designed such that if both transistors operate in the saturation region, their drain currents must meet the relationship $I_{3}<I_{4}\left(I_{5}>I_{6}\right)$. So $\mathrm{M}_{10}$ and $\mathrm{M}_{12}$ operate in the triode region such that voltages of node $\mathrm{N}_{1}$ and $\mathrm{N}_{2}$ are set to " 1 " and "0" to force transistors $\mathrm{M}_{13}$ and $\mathrm{M}_{16}$ to be turned off at steady state.

Once the load current decreases quickly and causes large output variations, the extra current $\Delta I_{6}$ is generated to pull the voltage of node $\mathrm{N}_{1}$ down. Then transistor $\mathrm{M}_{13}$ will then be heavily turned on to charge the gate capacitance of power transistor. When $V_{\text {OUT }}$ is regulated back to its expected voltage in the steady state, $I_{6}$ decreases and the voltage of node $\mathrm{N}_{1}$ is smoothly reset to " 1 " to turn transistor $\mathrm{M}_{13}$ off.

2) Sensitivity to Supply voltage: Similarly, the transistor $\mathrm{M}_{16}$ can be turned on by pulling the voltage of node $\mathrm{N}_{2}$ high to discharge the gate capacitance during the negative slewing period. As shown in Fig. 3(b), the traditional method to increase a PMOS current $I_{3}$ momentarily is just by pulling down the gate voltage directly [14]. However, there exist some problems for this structure. First, additional high-pass filter devices $\left(R_{2}\right.$ and $\left.C_{2}\right)$ are needed, occupying large chip area inevitably. Second, it is sensitive to supply voltage variations. This is because when a large coupling capacitor $C_{2}$ has been connected to the gate of $\mathrm{M}_{9}$ in Fig. 3(b), the bandwidth of current mirror $\mathrm{M}_{8}-\mathrm{M}_{9}$ is degraded due to the largely increased capacitance at the gate. 
As a result, the gate voltage $V_{G 9}$ cannot follow variations of the supply voltage in lowpower design. For example, when $V_{I N}$ increases fast, $V_{G S 9}$ may be enlarged instantaneously. $I_{3}$ is thus increased, having the potential risk to pull the node voltage $V_{\mathrm{N} 2}$ high and turn on $\mathrm{M}_{16}$ falsely. An unwanted discharging current $I_{\text {discharge }}$, depending on the amplitude of $\Delta V_{I N}$, flows to the gate of power MOS and tries to pull $V_{G}$ down. So the output voltage of the LDO is increased and must be regulated by the negative feedback loop in a certain time. Moreover, if $V_{I N}$ decreases rapidly, $\mathbf{M}_{9}$ may be shut down to delay the pull function of SRE circuit, because only a small bias current is used here to pull $V_{G 9}$ low. The PSRR and line-transient performances are therefore degraded, which has a similar phenomenon in [14].

The simplest solution is with the help of an RC filter in line with the power supply to filter out fluctuations before they reach the SRE circuit. However, the high power losses and reduction in voltage headroom caused by this resistor when the SRE circuit is triggered would severely limit its size, pushing the pole to high frequencies. Another methodology is assisted by adding a cascode structure such as resistors into the PMOS bias current pair $\mathrm{M}_{8}-\mathrm{M}_{9}$, making the coupling circuit insensitive to supply voltage noise. However, this may reduce the transconductance at the coupling input port and the dynamic charging effect is weakened. The new idea proposed in this brief to avoid such problems is just by adopting current subtracter $\mathrm{M}_{4}-\mathrm{M}_{6}$ instead as shown in Fig. 3(a). After that optimization, only one coupling capacitor $C_{1}$ is needed leading to a smaller chip area. The main difference compared to using two coupling capacitors is that the circuit speed response for enabling charging action is degraded by additional two current mirrors $\left(\mathrm{M}_{6}-\mathrm{M}_{7}\right.$ and $\left.\mathrm{M}_{8}-\mathrm{M}_{9}\right)$ and small bias current $I_{B}$. However, by making the sizes of these transistors small, the parasitic capacitances are set small and the time delay can be ignored. In addition, transistors $\mathbf{M}_{14}$ and $\mathbf{M}_{15}$ are used to prevent the noise of $\mathrm{N}_{1}$ and $\mathrm{N}_{2}$ from coupling to the gate of the power transistor when transistors $\mathrm{M}_{13}$ and $\mathrm{M}_{16}$ are turned on.

3) Optimal Sizing of Drive Transistors: The response time of the SRE circuit is determined by the time required to turn on or turn off drive transistors $\mathbf{M}_{13}$ and $\mathbf{M}_{16}$ when an output voltage spike $\Delta V$ is applied to the DPP SRE circuit. During the positive (negative) output slewing, transistor $\mathrm{M}_{12}\left(\mathrm{M}_{10}\right)$ is in the saturation region. Therefore, the response time $t_{r e s, p}$ and $t_{r e s, n}$ of the SRE circuit for positive and negative slewing periods is approximately given by

$$
\begin{aligned}
& t_{r e s, p} \approx \frac{\left(\left|V_{t h p}\right|-\left|V_{o v, M 12}\right|\right) C_{p 1}}{g_{m 11} \Delta V} \\
& t_{r e s, n} \approx \frac{\left(V_{t h n}-V_{o v, M 10}\right) C_{p 2}}{b_{1} g_{m 4} \Delta V}
\end{aligned}
$$

where $V_{o V}$ is the overdrive voltage of MOS transistor, $C_{p 1}\left(C_{p 2}\right)$ is the parasitic capacitance at node $\mathrm{N}_{1}\left(\mathrm{~N}_{2}\right)$. Equations (4) and (5) show that the response time increases with the value of $C_{p 1}$ and $C_{p 2}$. Increasing the sizes of transistors $\mathrm{M}_{13}$ and $\mathrm{M}_{16}$ thus slows down response time of the SRE circuit.

On the other hand, larger size of drive transistors is critical for controlling the amount of dynamic currents to charge and discharge $V_{G}$, therefore affecting the maximum attainable slew rate. For example, assuming that $\mathbf{M}_{13}$ and $\mathbf{M}_{16}$ are in 
saturation regions with constant dynamic current during the output slewing periods and the channel length modulation is neglected, the drive transistor size can then be demonstrated as

$$
\begin{aligned}
& \left(\frac{W}{L}\right)_{M 13} \approx \frac{2 \cdot S R_{+} \cdot C_{p a s s}}{u_{p} C_{o x}\left(V_{I N}-V_{o v, M 11}-\left|V_{t h p}\right|\right)^{2}} \\
& \left(\frac{W}{L}\right)_{M 16} \approx \frac{2 \cdot S R_{-} \cdot C_{p a s s}}{u_{n} C_{o x}\left(V_{I N}-\left|V_{o v, M 9}\right|-V_{t h n}\right)^{2}}
\end{aligned}
$$

where $S R_{+}$and $S R_{-}$are the slew rate improvement achieved by DPP SRE circuit. One method to resolve the trade-off between speed response and SR is to enlarge $g_{m 4}$ and $g_{m 11}$ without increasing much power.

From the above analysis, it seems that the dynamic push-pull scheme adds a gain into the loop by the boosted current and can effectively enhance the transient response time for regulating the output voltage back to a stable voltage level, i.e., the circuit is used to enhance the slew rate of the error amplifier during the transient period. Because the loop-bandwidth is low-pass and limited while this feedforward path due to capacitive coupling is high-pass, the values of $R_{1}$ and $C_{1}$ can thus be selected by setting the corner frequency $\left(1 / R_{1} C_{1}\right)$ a little lower than the GBW to extend the loopbandwidth of the LDO and make sure the DPP SRE circuit only works for high frequency spikes.

Comparisons between the loop gains of the proposed LDO and circuit structure like [13] at different load conditions are shown in Fig. 4 to prove the superiority. Method A (adaptive biasing) enlarges the bandwidth at heavy load and method B (dynamic push-pull techniques) focuses on voltage variation at high frequency, all of which will lead to a faster transient response at low bias current.

\section{Circuit Realization}

Fig. 5 shows the schematic of the proposed LDO regulator, which consists of a PMOS power transistor $\mathbf{M}_{\mathrm{o}}$, a current-sensing circuit, a high slew-rate push-pull error amplifier, a SRE circuit and a reference buffer. The push-pull output stage constructed with transistors $\mathrm{M}_{13}$ and $\mathrm{M}_{20}$ facilitates the LDO regulator using only moderate size $\mathrm{M}_{\mathrm{o}}$ to provide a wide range of load currents. In this circuit, to provide $100 \mathrm{~mA}$ load current with $150 \mathrm{mV}$ dropout, the aspect ratio of $(W / L)_{\mathrm{Mo}}$ is chosen to be $15000 \mu \mathrm{m} / 0.35 \mu \mathrm{m}$ in a $0.35 \mu \mathrm{m}$ standard CMOS process where the threshold voltage $\left|V_{\text {thp }}\right|$ of $\mathrm{M}_{\mathrm{o}}$ is about $0.66 \mathrm{~V}$.

The error amplifier is mainly constructed of two cross-coupled common-gate cells $G_{m H}$ and $G_{m L}$. Here some transistors like $\mathrm{M}_{2}$ and $\mathrm{M}_{3}$ have been reused in both input stages of the cells. The typical bandwidth of a LDO with $100 \mathrm{~mA}$ output capability is about $200 \mathrm{kHz}$ to $1 \mathrm{MHz}$ [1]-[3]. Assuming the corner frequency is set to be about 


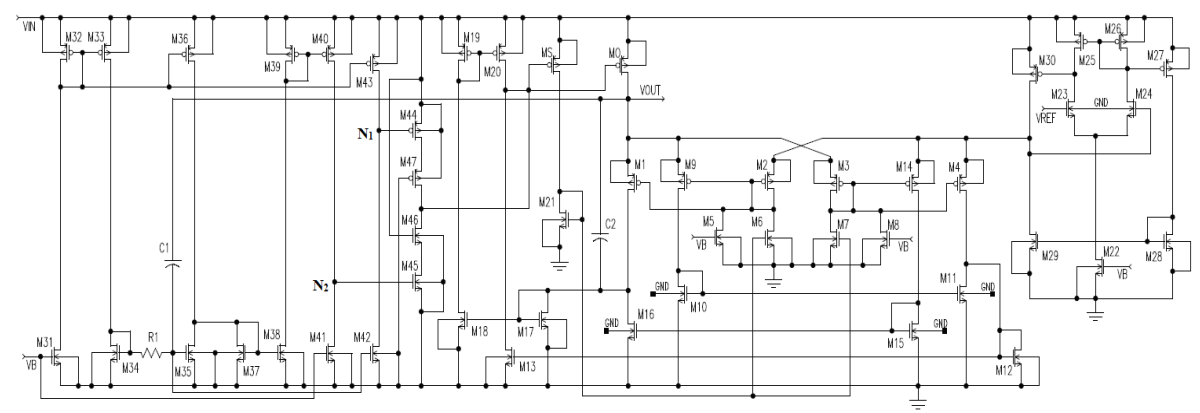

Fig. 5. The proposed ultra-fast capacitor-less LDO with a reference buffer

$100 \mathrm{kHz}$, the required passive components $R_{1}$ and $C_{1}$ are $3 \mathrm{pF}$ and $500 \mathrm{k} \Omega$ respectively, where the accuracy is not important. Current mirrors $\mathbf{M}_{12}-\mathrm{M}_{13}$ and $\mathbf{M}_{19}-\mathrm{M}_{20}$ are used to realize a current-summation circuit. To ensure the amplifier has fast-transient responses and large voltage-gain, the channel lengths of all transistors except $\mathrm{M}_{\mathrm{o}}$ are designed to be five times of the minimum feature size to guarantee that all the parasitic poles are at high frequency.

Since most of voltage references do not have output current driving ability [24], a voltage buffer without frequency compensation is introduced here to transfer the voltage $V_{R E F}$ to the inputs of the $\mathrm{G}_{\mathrm{m}}$ cells. Because adaptive biasing is utilized in the circuit, the resulting bias current for the amplifier is increased at heavy load, which requires an enhanced driving current $I_{\text {drive }}$ from the buffer. Therefore, the aspect ratio $(W / L)_{\mathrm{M} 30}$ should be designed to satisfy the maximum driving ability without using a large overdrive voltage, which must be always smaller than the dropout voltage at different loads. Otherwise, $\mathrm{M}_{30}$ may enter into linear region when the difference between $V_{I N}$ and $V_{\text {OUT }}$ is small. This will provide a low-resistance path where the supply noises can couple to inputs of the $G_{m}$ cells, degrading PSRR greatly. Furthermore, the bandwidth of the buffer should be designed to satisfy the maximum bias current variation rate when the load current changes from light load to heavy load. If not it will cause large voltage droop at the output of the buffer, deteriorating transient responses.

As shown in Fig. 5, because the output of the LDO is connected to a low-resistance node such as the source terminals of $\mathrm{M}_{1}$ and $\mathrm{M}_{3}$ inside the $G_{m H}$ cell, this sets the dominant pole $p_{1}$ locating at the gate of the power transistor and the output pole $p_{2}$ of the LDO to be non-dominant. As both drive transistors $\mathrm{M}_{44}$ and $\mathrm{M}_{45}$ are off in the LDO during the static state, there is almost no difference between the ac responses of the LDO with and without the SRE circuit. Here four parts mainly contribute to total output load capacitance $C_{\text {load }}$ in this structure, including $C_{d b}$ of the power MOSFET, input capacitors $C_{i n}$ from $G_{m}$ cells, coupling capacitor $C_{1}$ from DPP SRE circuit and the parasitic output capacitance $C_{\text {par }}$ due to the metal lines for on-chip power distribution which is generally in the range of 10-100pF [9]. By using the circuit proposed above, more input transistors and capacitors are implanted at the output of the LDO compared to [13], $C_{\text {load }}$ is therefore unluckily increased pushing $p_{2}$ to lower frequency, which may degrade phase margin of the feedback loop. This stability may be even worse when a large parasitic capacitance $C_{p a r}$ and small $I_{\text {load }}$ are applied [20]. 
In order to realize pole splitting under a wide range of $I_{\text {load }}$ from several tens of milliamperes to several $\mu \mathrm{A}$ and occupy less silicon area, the active capacitor multiplier is adopted for Miller compensation [25]. Here, capacitor $C_{2}$ performs the multiplied-miller capacitor with current buffer. The overall equivalent miller capacitor $C_{c}$ is equal to $k C_{2}$, where $k=\left(S_{18} / S_{17}\right) \times\left(S_{20} / S_{19}\right)$ and $S_{\mathrm{i}}=(W / L)_{\mathrm{i}}$ is the aspect ratio of the $\mathrm{i}$-th transistor.

Assuming $G_{m 1}$ and $R_{\mathrm{o} 1}$ are the equivalent first stage transconductance and output resistance of the LDO, $g_{m o}$ and $C_{\text {pass }}$ are the transconductance and gate capacitance of the power transistor, $R_{\text {out }}$ is the overall output resistance, the frequency response can then be given by

$$
\begin{gathered}
p_{1}=1 /\left[\left(C_{\text {pass }}+C_{c} g_{m o} R_{\text {out }}\right) R_{\text {o1 }}\right] \\
p_{2}=\left(g_{m o}+1 / R_{\text {out }}\right) /\left(C_{\text {load }}+C_{\text {pass }}\right) \propto \sqrt{I_{\text {load }}} \\
G B W=g_{m o} /\left[C_{\text {pass }} /\left(G_{m 1} R_{\text {out }}\right)+C_{c} g_{m o} / G_{m 1}\right] \propto \sqrt{I_{\text {load }}}
\end{gathered}
$$

Here the input resistance $1 / G_{m 1}$ of the error amplifier mainly determines $R_{\text {out }}$. Because adaptive biasing is applied, poles and GBW are changed accordingly in different load conditions as shown in equations (9) and (10). In order to make sure a phase margin larger than $45^{\circ}, p_{2}$ should be larger than GBW to determine the total Miller capacitor $C_{c}$.

$$
C_{c}>\frac{G_{m 1} R_{\text {out }}}{1+g_{m o} R_{\text {out }}}\left(C_{\text {load }}+C_{\text {pass }}\right)-\frac{C_{\text {pass }}}{g_{m o} R_{\text {out }}} \simeq \frac{G_{m 1} C_{\text {load }}}{g_{m o}}
$$

The minimum load current for the LDO to ensure stability is $0.5 \mathrm{~mA}$. Normally, the parameter $g_{m o} R_{\text {out }}$ or $g_{m o} / G_{m 1}$ is set large enough to make this compensation achieved without using any large on-chip compensation capacitors. In this design, the required compensation capacitor $C_{2}$ is only $2.3 \mathrm{pF}$. Area efficiency of such LDO regulator is thus maintained, which is particularly suitable for chip-level power management. Also the capacitor multiplier introduces a left-hand plane zero $z_{1}\left(g_{m 17} / C_{2}\right)$ at a relatively high frequency, which can be designed near the output pole $p_{2}$ to add phase and optimize frequency compensation.

\section{$4 \quad$ Experimental Results and Discussion}

The proposed capacitor-less LDO has been implemented in standard 0.35 $\mathrm{m}$ CMOS technology. The circuit was designed to provide $2.5 \mathrm{~V}$ output voltage at $100 \mathrm{~mA}$ output current for input voltage greater than $2.65 \mathrm{~V}$.

Load transient behavior, which is mainly decided by SR and its bandwidth of the LDO, is simulated here at to evaluate the transient performance. Fig. 6 shows the load-transient responses with a $100 \mathrm{pF}$ off-chip output capacitor, which is used to 


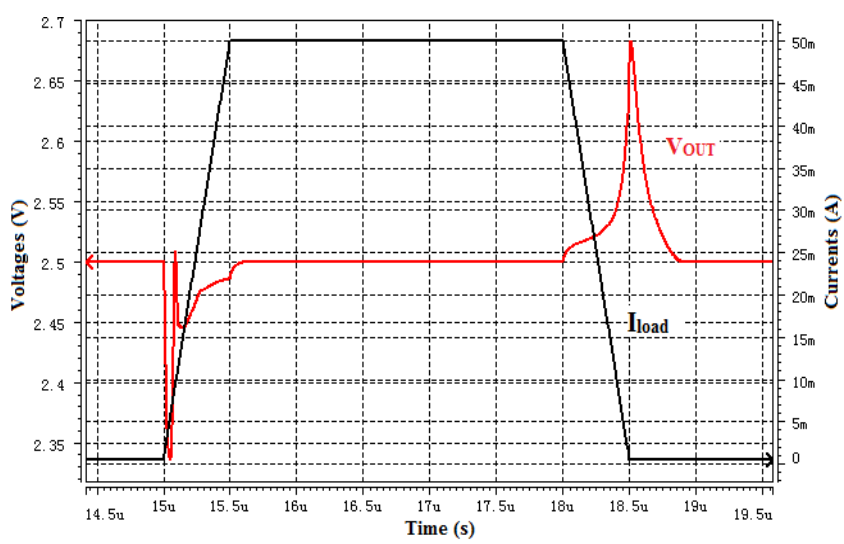

(a)
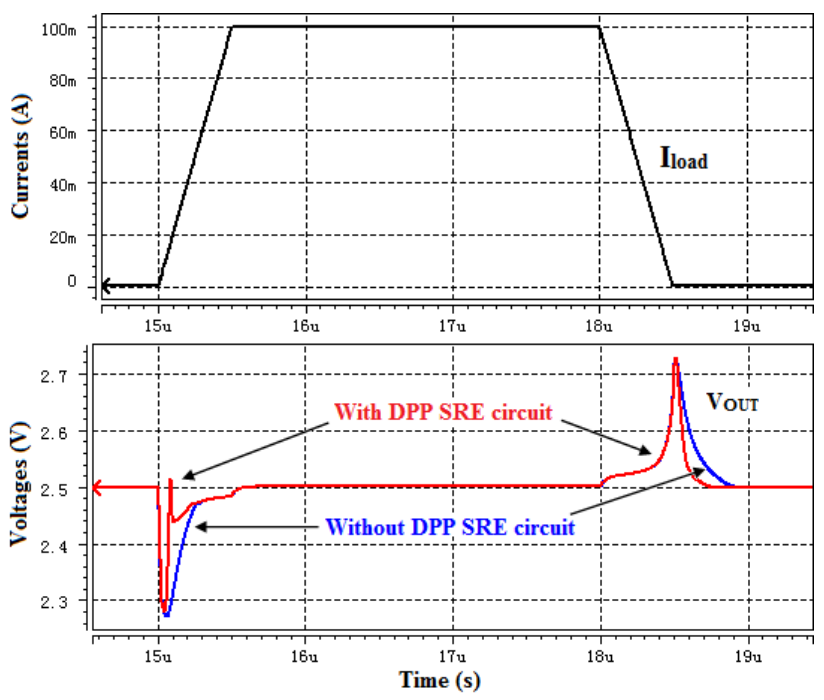

(b)

Fig. 6. Simulated load-transient responses with a $100 \mathrm{pF}$ off-chip output capacitor for different current changes (a) $\Delta \mathrm{I}_{\text {load }}=50 \mathrm{~mA}$ (b) $\Delta \mathrm{I}_{\text {load }}=100 \mathrm{~mA}$

model the output-parasitic capacitance from the metal lines. In Fig. 6(a), Iload varies from $0.5 \mathrm{~mA}$ to $50 \mathrm{~mA}$ and $V_{I N}$ is $2.8 \mathrm{~V}$, while the load current change is increased to $100 \mathrm{~mA}$ in Fig. 6(b). The results show that the output voltage can be fully recovered within $0.7 \mu$ s at a voltage spike less than $229 \mathrm{mV}$. It can be also observed in Fig. 6(b) that with the use of DPP SRE circuit in the LDO, which only consumes $29.4 \%$ additional static current, significant improvement in transient responses can be achieved in low-power condition. 


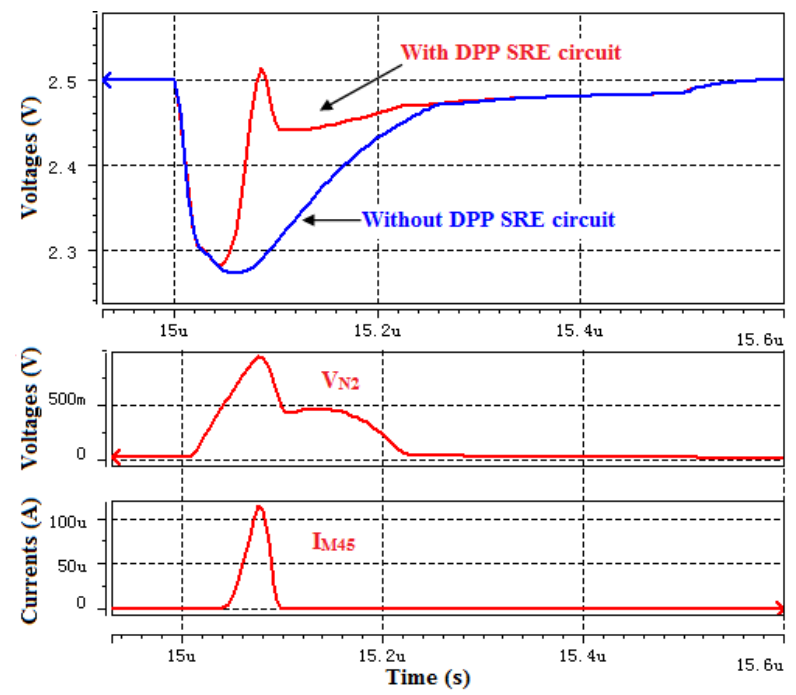

(a)
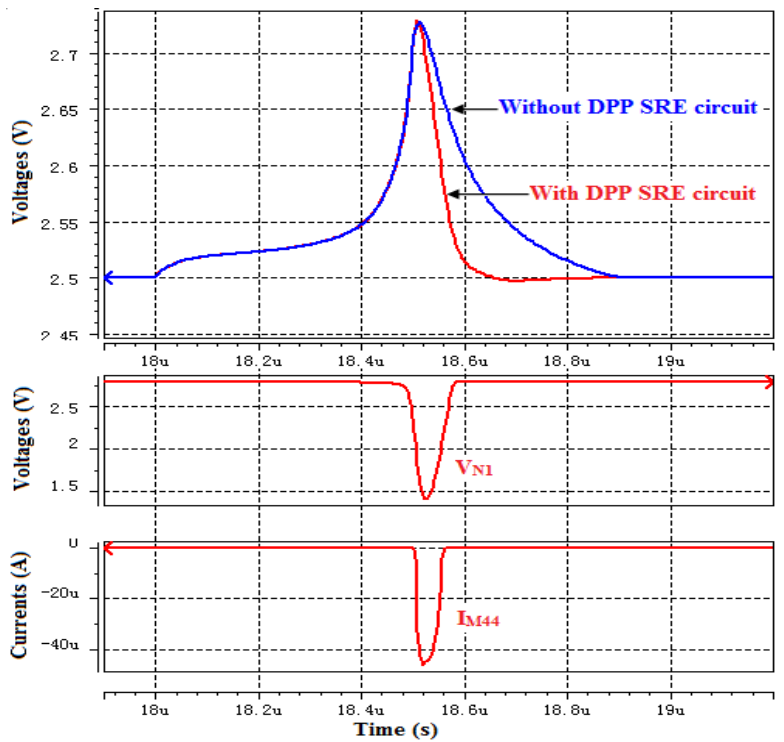

(b)

Fig. 7. Partial enlargement of the voltage spikes for a $100 \mathrm{pF}$ off-chip output capacitor and $100 \mathrm{~mA}$ load current change (a) undershoot (b) overshoot

The working process and advantage of the DPP SRE circuit can be analyzed in detail in Fig. 7. For example, when the load current changes from the light load to heavy load, a large undershoot occurs at the output. The current comparator $\left(\mathrm{M}_{40} / \mathrm{M}_{41}\right)$ takes effect to pull the node voltage $V_{\mathrm{N} 2}$ high and turn on drive transistor $\mathrm{M}_{45}$ heavily. A dynamic current $I_{\mathrm{M} 45}$ (more than $100 \mu \mathrm{A}$ ) can be provided to reduce the 
output-voltage excursion during transient. In addition, the pole located at the gate of the power transistor will be shifted to a higher frequency due to the turn on of drive transistor $\mathrm{M}_{45}$ during transient, thereby improving the bandwidth of the LDO. The settling time is therefore improved by about 2.9 times when a $100 \mathrm{~mA}$ output-current change is applied. The phenomenon for the overshoot reduction is similar in Fig. 7(b).

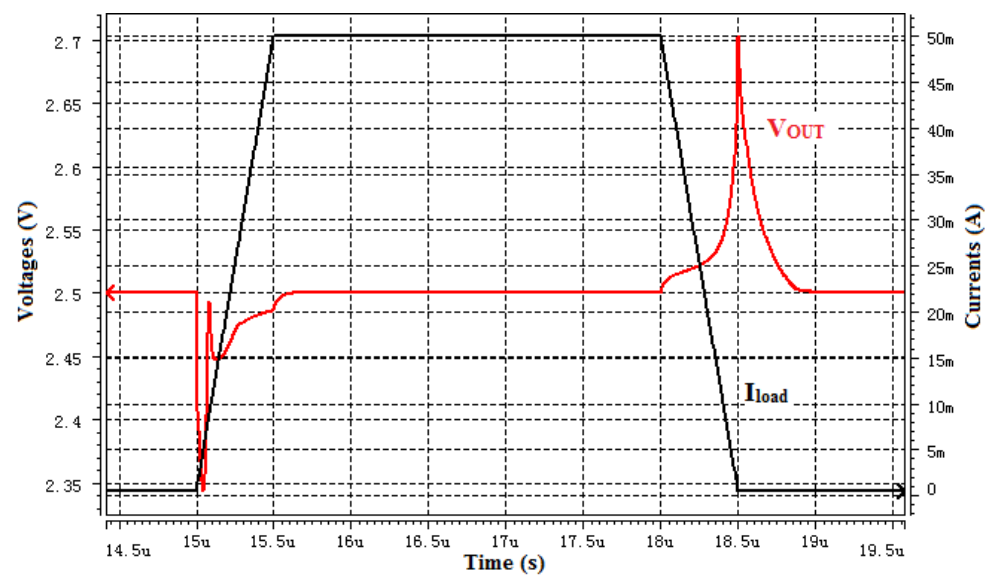

(a)

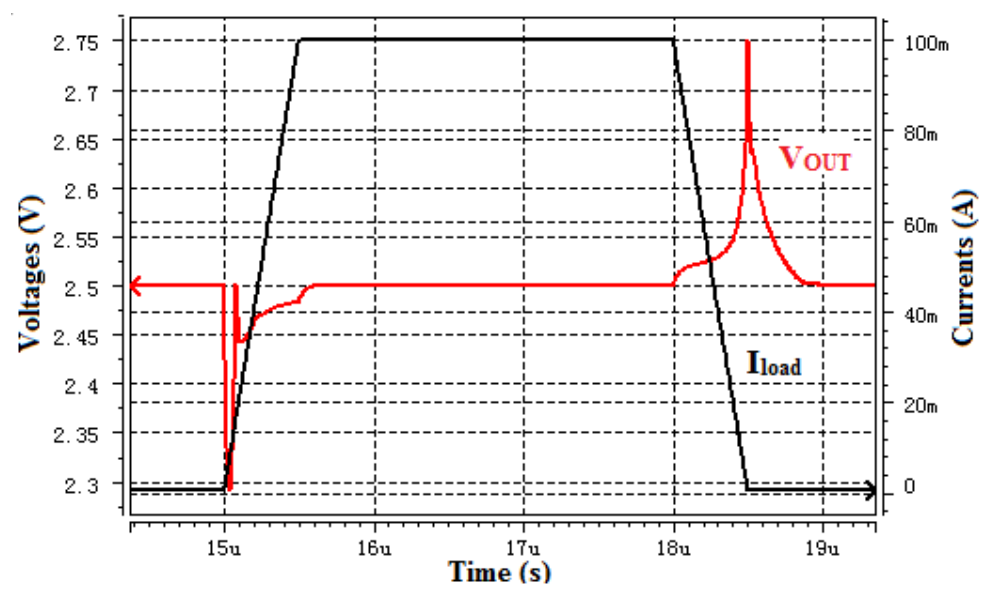

(b)

Fig. 8. Simulated load-transient responses without an off-chip output capacitor for different current changes (a) $\Delta \mathrm{I}_{\mathrm{load}}=50 \mathrm{~mA}$ (b) $\Delta \mathrm{I}_{\mathrm{load}}=100 \mathrm{~mA}$

The load-transient responses without an off-chip output capacitor are shown in Fig. 8 . The current change $\Delta I_{\text {load }}$ are chosen as $50 \mathrm{~mA}$ and $100 \mathrm{~mA}$ again respectively. Measurement results show that the proposed capacitor-less LDO can be fully recovered within $0.8 \mu \mathrm{s}$ at a voltage spike less than $250 \mathrm{mV}$. 
From the simulation results shown above, it can be seen that the DPP SRE circuit aids in adjusting the power MOS in the transient response effectively to avoid large output spikes, where the voltage deviation and response time are much better than that in [13] even at a twice load current change. The sizes of driving transistors in SRE circuit have been optimized to avoid overcharging at the gate of $\mathrm{M}_{\mathrm{o}}$ and good stability of the LDO regulator can be achieved.
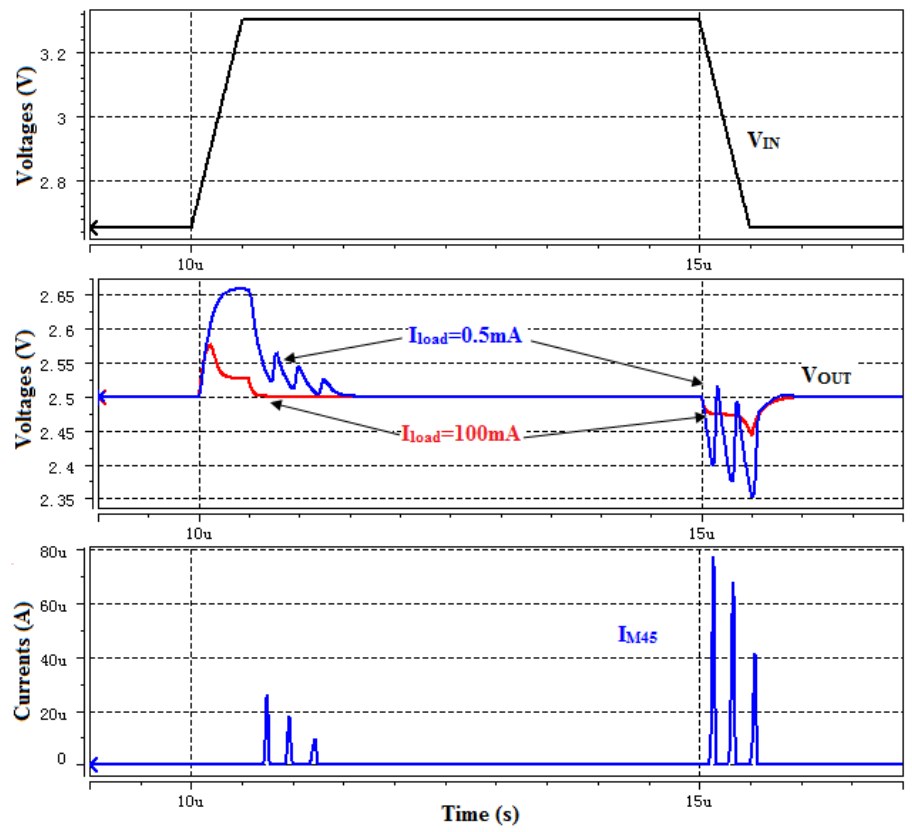

Fig. 9. Simulated line-transient response with a $100 \mathrm{pF}$ off-chip capacitor

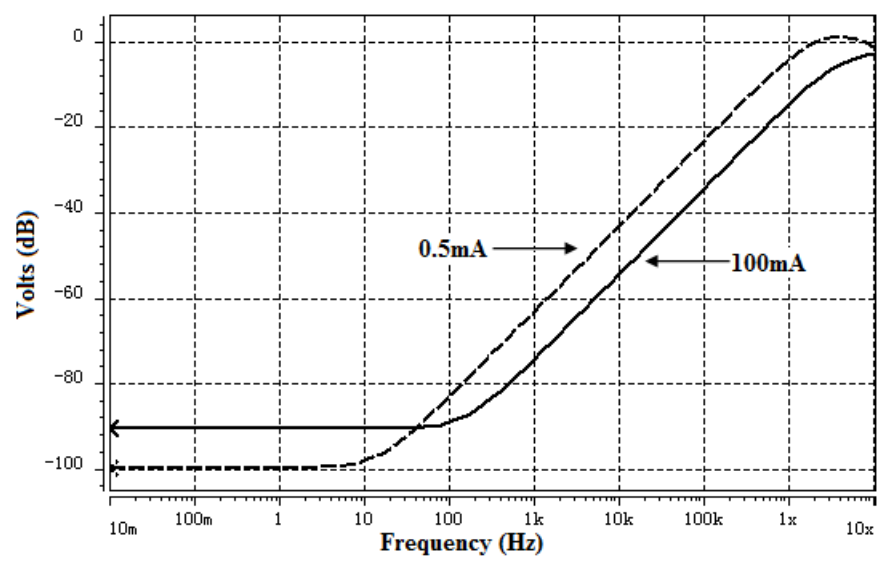

Fig. 10. Simulated PSRR versus frequency for $I_{\text {load }}=0.5 \mathrm{~mA}$ and $\mathrm{I}_{\mathrm{load}}=100 \mathrm{~mA}$ when $\mathrm{V}_{\mathrm{REF}}=2.5 \mathrm{~V}$, $\mathrm{V}_{\mathrm{IN}}=2.8 \mathrm{~V}$ and $\mathrm{C}_{\text {load }}=100 \mathrm{pF}$ 
To prove the DPP SRE circuit is not sensitive to supply voltage variations because of a current subtracter introduced, the line-transient is therefore simulated. The linetransient response with a $100 \mathrm{pF}$ off-chip output capacitor is shown in Fig. 9, where $V_{I N}$ varies from $2.65 \mathrm{~V}$ to $3.3 \mathrm{~V}$. The result shows that the output voltage can be fully recovered within $1 \mu$ s at a voltage spike less than $76 \mathrm{mV}$ when $I_{\text {load }}$ is $100 \mathrm{~mA}$. No dynamic spurs and injected noise occur and the circuit works robustly. However, if the load current is small, the bandwidth of the LDO is decreased, which may introduce a larger voltage spike and trigger the pull function of the DPP SRE circuit in a certain time. Because Miller compensation is adopted to guarantee sufficient phase margin during transient as analyzed before, the small ringing can be attenuated quickly without affecting the transient performance. Finally, power-supply ripple rejection (PSRR) against different load currents is shown in Fig. 10. The proposed LDO can achieve about $74 \mathrm{~dB}$ PSRR at $1 \mathrm{kHz}$ when $I_{\text {load }}=100 \mathrm{~mA}$.

Table 1. Performance Comparison With Previous Published Work

\begin{tabular}{|cccccc|}
\hline & {$[9]$} & {$[13]$} & {$[14]$} & {$[20]$} & This Work \\
\hline Technology $(\mu \mathrm{m})$ & 0.09 & 0.18 & 0.35 & 0.35 & 0.35 \\
\hline Input voltage $\mathrm{V}_{\mathrm{IN}}(\mathrm{V})$ & 1.2 & $1-1.8$ & $0.95-1.4$ & $3-4.2$ & $2.65-3.3$ \\
\hline Dropout voltage $\mathrm{V}_{\mathrm{DO}}(\mathrm{mV})$ & 300 & 100 & 200 & 200 & 150 \\
\hline Quiescent current $\mathrm{I}_{\mathrm{Q}}(\mu \mathrm{A})$ & 6000 & 1.2 & 43 & 65 & 10 (no load) \\
\hline Output current $\mathrm{I}_{\text {load }}(\mathrm{mA})$ & 100 & 50 & 100 & 50 & 100 \\
\hline Load regulation $(\mathrm{mV} / \mathrm{mA})$ & N/A & 0.148 & $\sim 0.4$ & $\sim 0.56$ & 0.005 \\
\hline Line regulation $(\mathrm{mV} / \mathrm{V})$ & N/A & 3.625 & N/A & $\sim 23$ & 0.38 \\
\hline Settling time $\mathrm{T}_{\text {settle }}(\mu \mathrm{s})$ & 0.00054 & $\sim 2.8$ & $\sim 3$ & $\sim 15$ & $\sim 0.5$ \\
\hline FOMM $(\mathrm{ns})$ & 0.032 & 0.067 & 1.29 & 19.5 & 0.05 \\
\hline
\end{tabular}

Table 1 shows performance comparison with some previously reported capacitorless LDOs. A figure of merit $\left(\mathrm{FOM}=T_{\text {settle }} \times I_{Q} / I_{\text {load(max) }}\right)$ used in [9] and [13] is adopted here to evaluate different current efficient designs for improving transient response. The response time $T_{\text {settle }}\left(T_{\text {settle }}=C_{\text {load }} \times \Delta V_{\text {OUT }} / I_{\text {load,max }}\right)$ is found from $C_{\text {load }}$ for a specified $I_{\text {load,max }}$ and $\Delta V_{\text {OUT }}$. A lower FOM implies a better slewing performance, where the proposed regulator has the lowest FOM $(0.05 \mathrm{~ns})$. This feature is very important and attractive to any high-density SoC applications.

\section{Conclusion}

This chapter presents an ultra-fast, capacitor-less LDO with an advanced commongate error amplifier and DPP SRE circuit. Some low-power methods like adaptive biasing and capacitive coupling have been adopted to improve both ICMR and loop bandwidth of the error amplifier greatly, while maintaining the traditional advantages such as low quiescent current and small chip area. By applying them to a LDO with a power-efficient methodology, the accuracy and response speed are significantly enhanced. The experimental results confirm that overshoots and undershoots in load transient of the LDO are improved greatly as results from the loop-gain-bandwidth enhancement. The performances are especially encouraging in chip-level power management. 


\section{References}

1. Rincon-Mora, G.A., Allen, P.E.: A low-voltage, low quiescent current, low drop-out regulator. IEEE J. Solid-State Circuits 33(1), 36-44 (1998)

2. Leung, K.N., Mok, P.K.T.: A capacitor-free CMOS low-dropout regulator with dampingfactor-control frequency compensation. IEEE J. Solid-State Circuits 38(10), 1691-1702 (2003)

3. Lau, S.K., Mok, P.K.T., Leung, K.N.: A low-dropout regulator for SoC with Q-reduction. IEEE J. Solid-State Circuits 42(4), 658-664 (2007)

4. Man, T.Y., Leung, K.N., Leung, C.Y., Mok, P.K.T., Chan, M.: Development of singletransistor-control LDO based on flipped voltage follower for SoC. IEEE Trans. Circuits Syst. I, Reg. Papers 55(5), 1392-1401 (2008)

5. Lin, H.-C., Wu, H.-H., Chang, T.-Y.: An active-frequency compensation scheme for CMOS low-dropout regulators with transient-response improvement. IEEE Trans. Circuits Syst. II, Exp. Briefs 55(9), 853-857 (2008)

6. Ho, M., Leung, K.N., Mak, K.-L.: A low-power fast-transient 90-nm low-dropout regulator with multiple small-gain stages. IEEE J. Solid-State Circuits 45(11), 2466-2475 (2010)

7. Lin, C.-H., Chen, K.-H., Huang, H.-W.: Low-Dropout Regulators With Adaptive Reference Control and Dynamic Push-Pull Techniques for Enhancing Transient Performance. IEEE Trans. Power Electron. 24(4), 1016-1022 (2009)

8. Lee, H., Mok, P.K.T., Leung, K.N.: Design of low-power analog drivers based on slewrate enhancement circuits for CMOS low-dropout regulators. IEEE Trans. Circuits Syst. II, Exp. Briefs 52(9), 563-567 (2005)

9. Hazucha, P., Karnik, T., Bloechel, B.A., Parsons, C., Finan, D., Borkar, S.: Area-efficient linear regulator with ultra-fast load regulation. IEEE J. Solid-State Circuits 40(4), 933-940 (2005)

10. Al-Shyoukh, M., Lee, H., Perez, R.: A transient-enhanced low-quiescent current lowdropout regulator with buffer impedance attenuation. IEEE J. Solid-State Circuits 42(8), 1732-1742 (2007)

11. Lam, Y.H., Ki, W.H.: A 0.9 V $0.35 \mu \mathrm{m}$ adaptively biased CMOS LDO regulator with fast transient response. In: Proc. IEEE Int. Solid-State Circuits Conf., pp. 442-626 (2008)

12. Zhan, C., Ki, W.H.: Output-capacitor-free adaptively biased low-dropout regulator for system-on-chips. IEEE Trans. Circuits and Systems I, Reg. Papers 57(5), 1017-1028 (2010)

13. Man, T.Y., Mok, P.K.T., Chan, M.: A high slew-rate push-pull output amplifier for lowquiescent current low-dropout regulators with transient-response improvement. IEEE Trans. Circuits Syst. II, Exp. Briefs 54(9), 755-759 (2007)

14. Or, P.Y., Leung, K.N.: An output-capacitorless low-dropout regulator with direct voltagespike detection. IEEE J. Solid-State Circuits 45(2), 458-466 (2010)

15. Guo, J.P., Leung, K.N.: A 6- $\mu \mathrm{W}$ chip-area-efficient output-capacitorless LDO in 90-nm CMOS technology. IEEE J. Solid-State Circuits 45(9), 1896-1905 (2010)

16. Zheng, C., Ma, D.: Design of monolithic CMOS LDO regulator with $\mathrm{D}^{2}$ coupling and adaptive transmission control for adaptive wireless powered bio-implants. IEEE Trans. Circuits and Systems I, Reg. Papers 58(10), 2377-2387 (2011)

17. Ho, M., Leung, K.N.: Dynamic bias-current boosting technique for ultralow-power lowdropout regulator in biomedical applications. IEEE Trans. Circuits Syst. II 58(3), 174-178 (2011) 
18. Ho, E.N.Y., Mok, P.K.T.: A capacitor-less CMOS active feedback low-dropout regulator with slew-rate enhancement for portable on-chip application. IEEE Trans. Circuits Syst. II, Exp. Briefs 57(2), 80-84 (2010)

19. Leung, K.N., Ng, Y.S.: A CMOS low-dropout regulator with a momentarily current-boosting voltage buffer. IEEE Trans. Circuits and Systems I, Reg. Papers 57(9), 2312-2319 (2010)

20. Milliken, R.J., Silva-Martínez, J., Sánchez-Sinencio, E.: Full on-chip CMOS low-dropout voltage regulator. IEEE Trans. Circuits and Systems I, Reg. Papers 54(9), 1879-1890 (2007)

21. Ramírez-Angulo, J.: A novel slew-rate enhancement technique for one stage operational amplifiers. In: Proc. IEEE Midwest Symp. Circuits and Systems, Ames, IA, pp. 11-13 (1996)

22. Degrauwe, M.G., Rijmenants, J., Vittoz, E.A., Man, J.J.D.: Adaptive biasing CMOS amplifiers. IEEE J. Solid-State Circuits SC-17(6), 522-528 (1982)

23. Nagaraj, K.: CMOS amplifiers incorporating a novel slew rate enhancement circuit. In: Proc. IEEE Custom Integrated Circuits Conf., pp. 11.6.1-11.6.5 (1990)

24. Ming, X., Ma, Y.-Q., Zhou, Z.-K., Zhang, B.: A high-precision compensated CMOS bandgap voltage reference without resistors. IEEE Trans. Circuits Syst. II, Exp. Briefs 57(10), 767-771 (2010)

25. Rincon-Mora, G.A.: Active capacitor multiplier in miller-compensated circuits. IEEE J. Solid-State Circuits 35(1), 26-32 (2000) 